\title{
Advancing Our Understanding of Self-harm, Suicidal Thoughts and Behaviours in Autism
}

\author{
Sarah A. Cassidy ${ }^{1}$ (D) Ashley Robertson ${ }^{2,4}$. Ellen Townsend ${ }^{1} \cdot$ Rory C. O'Connor $^{2} \cdot$ Jacqui Rodgers $^{3}$
}

Published online: 3 September 2020

○) Springer Science+Business Media, LLC, part of Springer Nature 2020

Until recently, self-harm, suicidal thoughts and behaviours have not received extensive attention in autism research (Cassidy 2020; Cassidy and Rodgers 2017). The extant research evidence indicates high rates of self-harm in autistic people, but this work has primarily focused on self-harm in the context of challenging and/or repetitive behaviour associated with intellectual disability (Minshawi et al. 2014). Although extremely important work, this research does not explore whether autistic people who self-harm also experience intent to end one's life, or whether self-harm increases risk of subsequent suicidal behaviours (as in the general population; Rebeiro et al. 2016). More recent research has explored self-harm and suicidality, as defined in the general population, in autistic people. Early work showed that $66 \%$ of adults recently diagnosed with Asperger Syndrome had contemplated suicide in their lifetime, and 35\% had planned or attempted suicide (Cassidy et al. 2014). Autistic adults are also significantly more likely to experience non-suicidal self-injury (NSSI) compared to the general population, and NSSI is associated with increased risk of suicidality in this group (Cassidy et al. 2018b; Maddox et al. 2017; Moseley et al. 2019, 2020). Large-scale population studies have followed, showing that autistic people are significantly more likely to die by self-harm and suicide compared to those in the general population (Hirvikoski et al. 2016; Hwang et al. 2019; Kirby et al. 2019).

Importantly, the majority of available research has focused on prevalence (Hedley and Uljarevic 2018). This has

Sarah A. Cassidy

Sarah.Cassidy@Nottingham.ac.uk

1 School of Psychology, University of Nottingham, Nottingham NG7 2RD, UK

2 Suicidal Behaviour Research Laboratory, Institute of Health \& Wellbeing, University of Glasgow, Glasgow, UK

3 Population Health Sciences Institute, Newcastle University, Newcastle, UK

4 School of Psychology, University of Glasgow, Glasgow, UK been instrumental in raising awareness of these critical life and death issues in the autistic community. However, there is relatively little research into why autistic people are at increased risk of self-harm and suicide, to inform treatment and prevention strategies (Cassidy 2020; Cassidy and Rodgers 2017). To make progress, it is crucial for different stakeholders to listen and learn from one another. This includes autism researchers, self-harm and suicide researchers, autistic people, and those who support them. Members of the guest editorial board for this special issue have developed international forums involving these stakeholder groups to support these efforts, including an international priority setting exercise which identified the top 10 autism community priorities for suicide prevention research (Cassidy et al. 2019b; Table 1). This special issue brings together a guest editorial team of autism, self-harm and suicide researchers, to enable researchers internationally to present scientific developments on the topic of self-harm, suicidal thoughts and behaviours in autistic people. Here we present the most recent advances in this important topic, and recommendations for future research in light of the autism community's priorities for suicide prevention.

\section{Assessment and Measurement}

Three papers explored assessment and measurement of selfharm, suicidal thoughts and behaviours in autistic people, in research and clinical practice-a top 10 community priority.

Jager-Hyman et al. (2020) surveyed 121 clinicians who felt significantly less confident in screening for suicide risk, and rated safety planning as significantly less acceptable, for their autistic compared to their non-autistic clients. Howe et al. (2020) conducted a systematic review of tools used to measure suicidality among children and youth with and without ASD diagnosis. Results showed that no suicidality assessment tool has yet been validated in autistic children and young people. Cassidy et al. (2020) explored whether a 
Table 1 Autism community's top 10 priorities for suicide prevention 2019 (from Cassidy et al. 2019b).

1. What barriers do autistic people experience when seeking help which may put them at greater risk of suicide?

2. What are the risk and protective factors for suicide in autism across the lifespan?

3. To what extent are autistic people not believed about the severity of their distress?

4. How can we further understand suicide where mental health is not a factor, across the lifespan?

5. How can we best identify and assess suicidal thoughts and suicidal behaviours in autistic people, in research and clinical practice?

6. How should interventions be adapted for autistic people and individual presentations?

7. What is the experience of suicidality in autistic people? Is this experience different to the general population?

8. How do autistic people seek help when they are in a crisis?

9. How well do existing models of understanding suicide apply to autistic people?

10. What is the impact of poor sleep on suicide risk in autistic people, and how can this be measured?

widely used suicidality assessment tool validated in the general population, (the Suicide Behaviours QuestionnaireRevised; SBQ-R, Osman et al. 2001), operates similarly in autistic compared to non-autistic adults. Analysis of online survey data in 371 participants showed that the structure of the SBQ-R was not equivalent in autistic compared to non-autistic adults. Autistic adults also reported difficulties interpreting and responding to the SBQ-R items, due to difficulties with abstract questions, complex language and response options, and lack of items relevant to autistic people's unique experience of suicidality.

Together these results further emphasise the crucial need for future research to identify the most effective ways of assessing self-harm, suicidal thoughts and behaviours in autistic people. This will enable crucial high-quality research to establish prevalence, understand risk and evaluate intervention.

\section{Prevalence and Risk Markers}

A group of papers focused on prevalence and broad risk markers for self-harm, suicidal thoughts and behaviours in autistic people-another community priority, underexplored in previous research.

Three papers explored both prevalence and risk markers for self-harm, suicidal thoughts and behaviours. Hand et al. (2019) report data from a large representative sample of 21,792 Medicare enrolled autistic adults, with or without co-occurring intellectual disability-4\% met criteria for suicidal ideation or suicide attempts/self-injury. Young age and co-occurring psychiatric conditions increased risk of suicide ideation and suicide attempts/self-injury. Co-occurring intellectual disability was associated with increased risk of suicide attempts/self-injury, but not of suicide ideation, which could indicate difficulties in assessment of suicide ideation in autistic with intellectual disability. Hunsche et al. (2020) report results from a longitudinal study of suicidal ideation, self-injury/suicidal behaviour in 178 autistic children aged
7-11 years. Parent reported suicidal ideation occurred in $9.6 \%$, and self-injury/suicidal behaviours in $14.6 \%$ of children using the Child Behaviour Checklist. Oliphant et al. (2020) report results from a systematic literature review exploring the prevalence of self-harm in autistic children and young people under 18 years. Only 9 studies were identified, with prevalence estimates of self-harm, suicidal ideation or suicidal behaviour ranging between 11 and 73\%. No studies had used a tool with evidence of validity to assess self-harm in autistic children or young people.

A number of papers explored whether known risk markers for suicide in the general population can help explain increased risk in autistic people. DiBlasi et al. (2020) report results from the first preliminary genetic study of suicidal behaviour in autistic people, suggesting overlap between the genes involved in autism and increased risk of suicidal behaviour. In 481 autistic youth, McDonnell et al. (2019) report correlates of suicidal ideation and self-harm/suicide behaviours, notably child age, parental education, restricted and repetitive behaviours, IQ and adaptive behaviour, affective and conduct problems, and medical concerns. Conner et al. (2020) showed significantly increased risk of suicidality in autistic youth from the community and psychiatric inpatient samples, and emotion regulation was associated with increased risk of suicidality. Licence et al. (2019) showed a $24 \%$ parent reported prevalence of self-harm in autistic children and adults. Low mood and overactivity/ impulsivity predicted the presence of self-harm, with the model correctly classifying a majority $(82.9 \%)$ of cases.

Hochard et al. (2020) report online survey data from 650 general population adults, assessing associations between autistic traits, sleep duration and suicidality. Results suggest that autistic traits and short sleep duration are separately associated with suicidal ideation, but do not interact. Costa et al. (2020) showed in 150 autistic and 189 non-autistic adults, self-reported autistic traits, depressive symptomatology, and antidepressant intake significantly predicted suicidality. In those with high autistic traits, alexithymia was associated with further increased risk of suicidality. Arwert 
and Sizoo (2020) showed in 75 autistic adults, self-esteem was significantly associated with current suicidal ideation, and rumination with history of suicide attempts. Neither self-esteem or rumination were significantly associated with severity of suicidality after controlling for depression. South et al. (2019) report on 74 women with significant social difficulties and high autistic traits. Self-reported imagination and repetitive behaviours were associated with suicidality, suggesting that feeling sad, alongside not being able to see a way out of one's current circumstances may increase risk of suicide in autistic people regardless of diagnostic status.

These studies suggest overlap between the characteristics of autism and risk markers for suicidality in autistic people, from the biological (DiBlasi et al. 2020) to the cognitive, affective and behavioural level. Studies identified common risk markers, such as low mood (e.g. Licence et al. 2019; South et al. 2019; Arwet and Sizoo 2020), age (Hand et al. 2019; McDonnell et al. 2019), repetitive behaviours and rumination (South et al. 2019; McDonnell et al. 2019; Arwet and Sizoo 2020). Other potential risk markers include impulsivity (Licence et al. 2019), alexithymia (Costa et al. 2020) and self-esteem (Arwet and Sizoo 2020). Many of these are common risk markers for suicide in the general population (O'Connor and Kirtely 2018). However, the characteristics of autism could amplify the risk. Increased tendency to perseverate on a particular train of thought or behaviour and difficulty imagining alternatives could increase risk of feeling entrapped with suicide perceived as the only possible escape route (South et al. 2019; Arwet and Sizoo 2020).

\section{Theoretical Models}

Two papers explored how a widely cited model of suicide - the Interpersonal Theory of Suicide (ITS)—could help explain increase risk of suicidality in autistic and nonautistic people. Pelton et al. (2020) showed that autistic people were significantly more likely to experience thwarted belonging and perceived burdensomeness compared to non-autistic people, and both mediated associations between autistic traits and suicidality. The predictions of the ITS were broadly upheld in both groups, but associations were significantly attenuated in autistic compared to non-autistic adults. Cassidy et al. (2019a) explored how the ITS could be expanded for autistic people, exploring the role of camouflaging one's autistic traits in experiences of thwarted belonging and suicidality. Results suggest that those with high autistic traits attempt to camouflage these in order to fit in in social situations, which is associated with increased feelings of thwarted belongingness and suicidality.

These studies show that there are likely unique as yet unknown risk markers for suicide in autistic people, which need to be included in existing suicide theories developed for the general population.

\section{Recommendations for Future Research}

This special issue represents a step change in our understanding of self-harm, suicidal thoughts and behaviours in autistic people, across childhood, adolescence and adulthood. However, there are clear avenues for future research.

First, it is crucial given the early stage of the research cycle, that we meaningfully involve autistic people and those who support them in developing high quality, useful and ethical self-harm and suicide research with and for autistic people. This special issue illustrates welcome overlap between the priorities of the autism community and research being undertaken, particularly in relation to understanding the best ways of assessing self-harm, suicidal thoughts and behaviours, identifying risk markers, exploring how suicide theories developed for the general population could apply to and be adapted for autistic people, and exploring the contribution of poor sleep to suicidality in autistic people. However, there was a lack of studies exploring other important aspects of suicidality, such as personalised interventions, and help seeking behaviour-only one study reported on the potential of suicide safety planning for autistic people from the perspective of clinicians (Jager-Hyman et al. 2020). Second, assessment and measurement of self-harm, suicidal thoughts and behaviours in autism are in their infancy (Howe et al. 2020; Cassidy et al. 2018a). It is essential to evaluate the most effective ways of identifying and assessing selfharm, suicidal thoughts and behaviours in future research and clinical practice. Third, research exploring self-harm, suicidal thoughts and behaviours in autism has tended not to be theoretically driven, and only two studies in this special issue explored how suicide theory in the general population could apply to autistic people (Cassidy et al. 2020; Pelton et al. 2020). Suicide models developed for the general population include many risk markers which are relevant to autistic people and identified in this special issue (e.g. social connections, rumination and impulsivity), and attempt to identify factors that distinguish those who contemplate suicide from those who go onto attempt suicide (Cassidy 2019a; O'Connor and Kirtley 2018). Self-harm and suicide research for autistic people needs to take these important next steps, in order to better understand and prevent suicide and reduce the distress experienced by autistic members of our community.

Acknowledgments We sincerely thank the authors and reviewers of all articles included in this special issue. We also extend our sincere thanks to the autism community, who have supported the research published in this special issue, and contributed to our understanding and prevention of self-harm and suicidality in autistic people. 
Author Contributions SAC wrote the first draft of the manuscript. All authors provided critical feedback on the draft manuscript and approved the final version for publication.

\section{References}

Arwert, T. G., \& Sizoo, B. B. (2020). Self-reported suicidality in male and female adults with autism spectrum disorders: Rumination and self-esteem. Journal of Autism and Developmental Disorders. https://doi.org/10.1007/s10803-020-04372-z.

Cassidy, S. (2020). Suicidality and self-harm in autism spectrum conditions. In S. White, B. Maddox, \& C. Mazefsky (Eds.), Oxford handbook of autism and co-occurring psychiatric conditions. Oxford: Oxford University Press.

Cassidy, S. A., Bradley, L., Bowen, E., Wigham, S., \& Rodgers, J. (2018a). Measurement properties of tools used to assess suicidality in autistic and general population adults: A systematic review. Clinical Psychology Review, 62, 56-70.

Cassidy, S. A., Bradley, L., Cogger-Ward, H., Shaw, R., Bowen, E., Glod, M., et al. (2020). Measurement properties of the suicidal behaviour questionnaire-revised in autistic adults. Journal of Autism and Developmental Disorders. https://doi.org/10.1007/ s10803-020-04431-5.

Cassidy, S., Bradley, P., Robinson, J., Allison, C., McHugh, M., \& Baron-Cohen, S. (2014). Suicidal ideation and suicide plans or attempts in adults with Asperger's syndrome attending a specialist diagnostic clinic: A clinical cohort study. The Lancet Psychiatry, 1(2), 142-147.

Cassidy, S., Bradley, L., Shaw, R., \& Baron-Cohen, S. (2018b). Risk markers for suicidality in autistic adults. Molecular Autism, 9(1), 42.

Cassidy, S. A., Gould, K., Townsend, E., Pelton, M., Robertson, A. E., \& Rodgers, J. (2019a). Is camouflaging autistic traits associated with suicidal thoughts and behaviours? Expanding the interpersonal psychological theory of suicide in an undergraduate student sample. Journal of Autism and Developmental Disorders. https:// doi.org/10.1007/s10803-019-04323-3.

Cassidy, S., \& Rodgers, J. (2017). Understanding and prevention of suicide in autism. The Lancet Psychiatry, 4(6), e11.

Cassidy, S. A., Cogger-Ward, H., Goodwin, J., Roberston, A., \& Rodgers, J. (2019b). Autism Community Priorities for Suicide Prevention. Retrieved from https://sites.google.com/view/mentalheal thinautism/projects/insar-policy-briefing.

Conner, C. M., Golt, J., Righi, G., Shaffer, R., Siegel, M., \& Mazefsky, C. A. (2020). A comparative study of suicidality and its association with emotion regulation impairment in large ASD and US census-matched samples. Journal of Autism and Developmental Disorders. https://doi.org/10.1007/s10803-020-04370-1.

Costa, A. P., Loor, C., \& Steffgen, G. (2020). Suicidality in adults with autism spectrum disorder: The role of depressive symptomatology, alexithymia, and antidepressants. Journal of Autism and Developmental Disorders. https://doi.org/10.1007/s10803-02004433-3.

DiBlasi, E., Kirby, A. V., Gaj, E., Docherty, A. R., Keeshin, B. R., Bakian, A. V., et al. (2020). Brief report: Genetic links between autism and suicidal behavior-A preliminary investigation. Journal of Autism and Developmental Disorders. https://doi. org/10.1007/s10803-020-04419-1.

Hand, B. N., Benevides, T. W., \& Carretta, H. J. (2019). Suicidal ideation and self-inflicted injury in medicare enrolled autistic adults with and without co-occurring intellectual disability. Journal of Autism and Developmental Disorders. https://doi.org/10.1007/ s10803-019-04345-x.
Hedley, D., \& Uljarević, M. (2018). Systematic review of suicide in autism spectrum disorder: Current trends and implications. Current Developmental Disorders Reports, 5(1), 65-76.

Hirvikoski, T., Mittendorfer-Rutz, E., Boman, M., Larsson, H., Lichtenstein, P., \& Bölte, S. (2016). Premature mortality in autism spectrum disorder. The British Journal of Psychiatry, 208(3), 232-238.

Hochard, K. D., Pendrous, R., Mari, T., \& Flynn, S. (2020). Examining the relationship between autism traits and sleep duration as predictors of suicidality. Journal of Autism and Developmental Disorders. https://doi.org/10.1007/s10803-020-04405-7.

Howe, S. J., Hewitt, K., Baraskewich, J., Cassidy, S., \& McMorris, C. A. (2020). Suicidality among children and youth with and without autism spectrum disorder: A systematic review of existing risk assessment tools. Journal of Autism and Developmental Disorders. https://doi.org/10.1007/s10803-020-04394-7.

Hunsche, M. C., Saqui, S., Mirenda, P., Zaidman-Zait, A., Bennett, T., Duku, E., et al. (2020). Parent-reported rates and clinical correlates of suicidality in children with autism spectrum disorder: A longitudinal study. Journal of Autism and Developmental Disorders. https://doi.org/10.1007/s10803-020-04373-y.

Hwang, Y. I., Srasuebkul, P., Foley, K. R., Arnold, S., \& Trollor, J. N. (2019). Mortality and cause of death of Australians on the autism spectrum. Autism Research, 12(5), 806-815.

Jager-Hyman, S., Maddox, B. B., Crabbe, S. R., \& Mandell, D. S. (2020). Mental health clinicians' screening and intervention practices to reduce suicide risk in autistic adolescents and adults. Journal of Autism and Developmental Disorders. https://doi. org/10.1007/s10803-020-04441-3.

Kirby, A. V., Bakian, A. V., Zhang, Y., Bilder, D. A., Keeshin, B. R., \& Coon, H. (2019). A 20-year study of suicide death in a statewide autism population. Autism Research, 12(4), 658-666.

Licence, L., Oliver, C., Moss, J., \& Richards, C. (2019). Prevalence and risk-markers of self-harm in autistic children and adults. Journal of Autism and Developmental Disorders. https://doi.org/10.1007/ s10803-019-04260-1.

McDonnell, C. G., DeLucia, E. A., Hayden, E. P., Anagnostou, E., Nicolson, R., Kelley, E., et al. (2019). An exploratory analysis of predictors of youth suicide-related behaviors in autism spectrum disorder: Implications for prevention science. Journal of Autism and Developmental Disorders. https://doi.org/10.1007/s1080 3-019-04320-6.

Maddox, B. B., Trubanova, A., \& White, S. W. (2017). Untended wounds: Non-suicidal self-injury in adults with autism spectrum disorder. Autism, 21(4), 412-422.

Minshawi, N. F., Hurwitz, S., Fodstad, J. C., Biebl, S., Morriss, D. H., $\&$ McDougle, C. J. (2014). The association between self-injurious behaviors and autism spectrum disorders. Psychology Research and Behavior Management, 7, 125.

Moseley, R. L., Gregory, N. J., Smith, P., Allison, C., \& Baron-Cohen, S. J. M. A. (2019). A 'choice', an 'addiction', a way 'out of the lost': Exploring self-injury in autistic people without intellectual disability. Molecular Autism, 10(1), 1-23.

Moseley, R. L., Gregory, N. J., Smith, P., Allison, C., \& Baron-Cohen, S. (2020). Links between self-injury and suicidality in autism. Molecular Autism, 11(1), 1-15.

O’Connor, R. C., \& Kirtley, O. J. (2018). The integrated motivationalvolitional model of suicidal behaviour. Philosophical Transactions of theRoyal Society B: Biological Sciences, 373(1754), 20170268.

Oliphant, R. Y., Smith, E. M., \& Grahame, V. (2020). What is the prevalence of self-harming and suicidal behaviour in under $18 \mathrm{~s}$ with ASD, with or without an intellectual disability? Journal of Autism and Developmental Disorders. https://doi.org/10.1007/ s10803-020-04422-6.

Osman, A., Bagge, C. L., Gutierrez, P. M., Konick, L. C., Kopper, B. A., \& Barrios, F. X. (2001). The Suicidal Behaviors 
Questionnaire-Revised (SBQ-R): Validation with clinical and nonclinical samples. Assessment, 8(4), 443-454.

Pelton, M. K., Crawford, H., Robertson, A. E., Rodgers, J., BaronCohen, S., \& Cassidy, S. (2020). Understanding suicide risk in autistic adults: Comparing the Interpersonal Theory of Suicide in autistic and non-autistic samples. Journal of Autism and Developmental Disorders. https://doi.org/10.1007/s10803-020-04393-8.

Ribeiro, J. D., Franklin, J. C., Fox, K. R., Bentley, K. H., Kleiman, E. M., Chang, B. P., et al. (2016). Self-injurious thoughts and behaviors as risk factors for future suicide ideation, attempts, and death: A meta-analysis of longitudinal studies. Psychological Medicine, 46(2), 225-236.
South, M., Beck, J. S., Lundwall, R., Christensen, M., Cutrer, E. A., Gabrielsen, T. P., et al. (2019). Unrelenting depression and suicidality in women with autistic traits. Journal of Autism and Developmental Disorders. https://doi.org/10.1007/s10803-019-04324-2.

Publisher's Note Springer Nature remains neutral with regard to jurisdictional claims in published maps and institutional affiliations. 